\title{
Patterns and Economic Burden of Hospitalizations and Exacerbations Among Patients Diagnosed with Idiopathic Pulmonary Fibrosis
}

\author{
Yanni F. Yu, DSc; Ning Wu, PhD; Chien-Chia Chuang, PhD; Rosa Wang, BS, MHA; Xiaoyun Pan, MS, PhD;
} Nicole N. Benjamin, BS; Giovanna Devercelli, PhD; and David B. Coultas, MD

\begin{abstract}
BACKGROUND: Idiopathic pulmonary fibrosis (IPF) is a rare and fatal restrictive respiratory disease under the idiopathic lung disease (ILD) class. IPF is a form of chronic, progressive fibrosing interstitial pneumonia and has more scarring, less inflammation, and poorer prognosis than most other ILD forms. Exacerbation of IPF is rapid, with unpredictable deterioration of lung function, and is associated with short-term mortality. The American Thoracic Society (ATS) evidence-based guidelines for diagnosis and management of IPF reports that the incidence of acute exacerbations is between $5 \%-10 \%$. Limited real-world evidence has been identified in the United States that assesses patterns of hospitalization, exacerbation of IPF, and the associated economic burden.
\end{abstract}

OBJECTIVES: To (a) characterize patients newly diagnosed with IPF and (b) examine incidence rates and costs of all-cause hospitalizations, IPF-related hospitalizations, and exacerbations.

METHODS: A retrospective analysis was performed with a national commercial claims database from calendar years 2006 to 2011. Newly diagnosed IPF patients were identified with either $\geq 2$ claims for idiopathic fibrosing alveolitis (IFA) or $\geq 1$ claim for IFA and $\geq 1$ claim for postinflammatory pulmonary fibrosis and a lung biopsy or thoracic high-resolution computed tomography within 90 days of the first claim for IFA (index date). IPF-related hospitalizations and possible IPF exacerbations were defined based on diagnoses recorded on event claims. Frequency, incidence rate, duration of events, and associated costs from the third-party payer's perspective were estimated.

RESULTS: Among 1,735 identified IPF patients, 38.6\% had at least 1 all-cause hospitalization; $10.8 \%$ had IPF-related hospitalizations; $4.6 \%$ had suspected IPF exacerbations leading to hospitalization; and $72.1 \%$ had suspected IPF exacerbations leading to urgent outpatient visits during the 1-year post-index period. Incident rates for these 4 events were 83 $(95 \% \mathrm{Cl}=79-88), 17(95 \% \mathrm{Cl}=14-19), 7(95 \% \mathrm{Cl}=6-9)$, and $277(95 \%$ $\mathrm{Cl}=269-286)$ per 100 person-years, respectively. Average costs per event were $\$ 13,987(S D=\$ 41,988), \$ 16,812(S D=\$ 66,399), \$ 14,731$ $(\mathrm{SD}=\$ 85,468)$, and $\$ 444(\mathrm{SD}=\$ 1,481)$, respectively.

CONCLUSIONS: Hospitalizations and possible exacerbations among patients with IPF were costly. Appropriate management of IPF needs to be considered to help slow IPF disease progression.

J Manag Care Spec Pharm. 2016;22(4):414-23

Copyright $\odot 2016$, Academy of Managed Care Pharmacy. All rights reserved.

\section{What is already known about this subject}

Compared with other idiopathic lung diseases, idiopathic pulmonary fibrosis (IPF) is characterized as a form of chronic, progressive fibrosing interstitial pneumonia that has more scarring, less inflammation, and unpredictable acute exacerbation. The annual incidence rate of acute respiratory worsening has been on the rise, with more IPF-related hospitalizations and IPF acute exacerbations.
Episodes of IPF acute exacerbation requiring hospitalization are associated with significant morbidity and mortality. In a 2007 review of acute exacerbations of IPF, Collard et al. proposed diagnostic criteria for exacerbations and emphasized the need for epidemiological data on exacerbations.

\section{What this study adds}

This is the first study to assess the economic burden of IPFrelated hospitalizations and exacerbations. The incidence rates were 7 or 277 events per 100 person-years for possible IPF exacerbations requiring or not requiring hospitalization, respectively. The estimated mean costs per event were $\$ 16,812$ and $\$ 14,731$ for IPF-related hospitalization and possible IPF exacerbation requiring hospitalization, respectively.

Hospitalizations and possible exacerbations among patients diagnosed with IPF were associated with high economic burden; effective treatments to manage IPF and reduce exacerbations are needed.

T diopathic pulmonary fibrosis (IPF) is a rare and fatal restrictive lung disease and the most common form among interstitial lung diseases. ${ }^{1}$ IPF has a variable clinical course, which includes rapid or slow disease progression as well as unpredictable acute exacerbation. ${ }^{2,3}$ Acute exacerbation of IPF is defined as rapid and unpredictable deterioration that is not due to infection, pulmonary embolism, or heart failure. ${ }^{4}$ Episodes of acute exacerbation requiring hospitalization are a grave concern, since it is associated with significant morbidity and mortality. 3,5 A randomized controlled trial in IPF showed that of 56 patients who were followed for 3 years, 57\% were rehospitalized for acute exacerbation, and 53\% of those patients eventually died. ${ }^{6}$

The estimated frequency of acute exacerbation varies because of variable definitions, diagnostic uncertainty, and difference in care settings, study designs, patient selection, or follow-up period. ${ }^{4,7}$ Moreover, it may be difficult to distinguish between acute respiratory worsening from an identifiable cause and acute exacerbations of IPF with unknown cause. The annual incidence of acute exacerbation among patients with IPF has been estimated at 5\%-10\%, with acute exacerbations comprising a subset of these episodes. ${ }^{7-10}$ Higher rates of acute 


\section{FIGURE 1 Sample Selection of Newly Diagnosed IPF Patients}

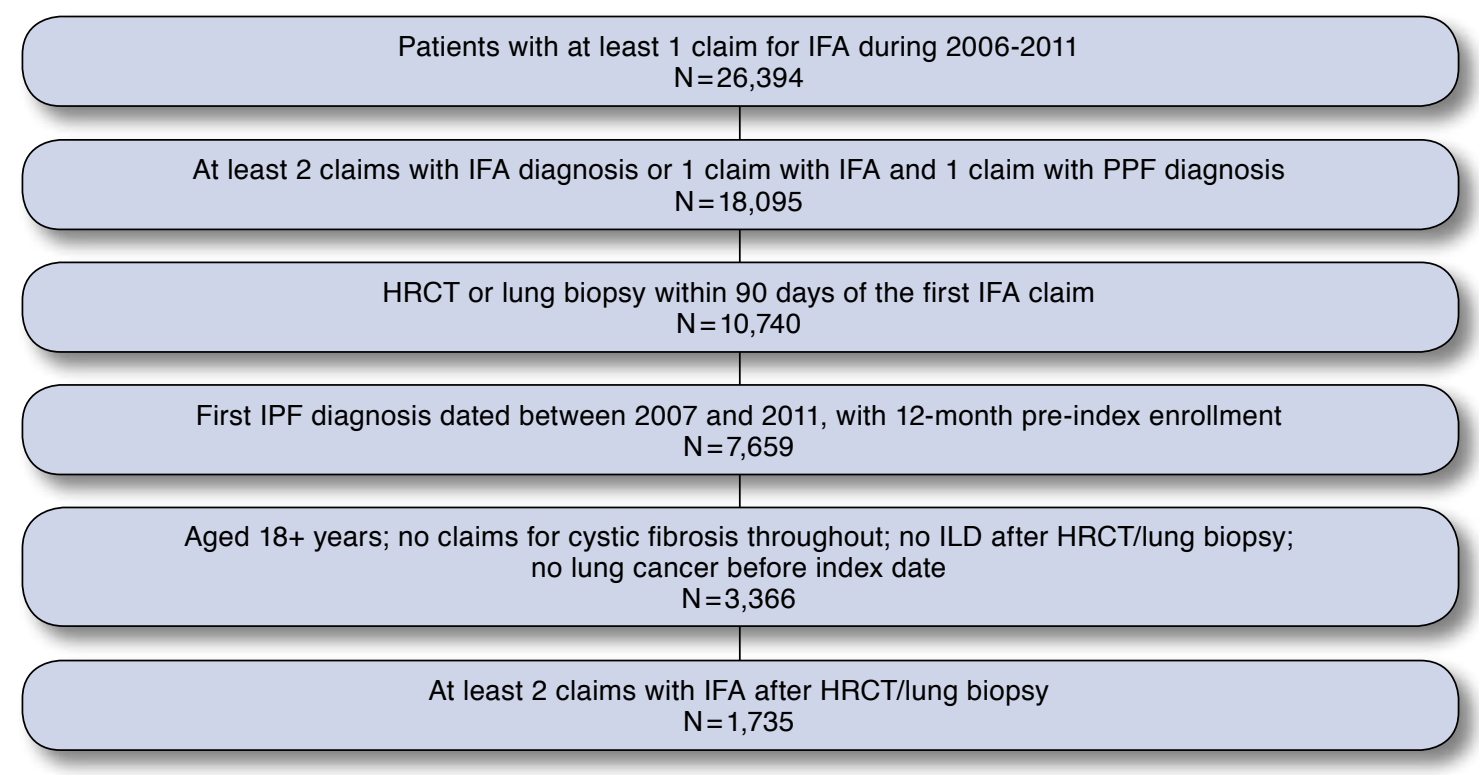

HRCT = high resolution computed tomography; IFA = idiopathic fibrosing alveolitis; ILD=idiopathic lung disease; IPF =idiopathic pulmonary fibrosis; $P P F=$ postinflammatory pulmonary fibrosis.

exacerbations have been reported in a clinical trial review study and several medical record review studies in Japan and South Korea. The 1-year and 3-year incidence rates of acute exacerbations were found to vary from $4.4 \%-19.0 \%$ and $20.7 \%-$ $23.9 \%$, respectively. ${ }^{11-13}$

In a 2007 review of acute exacerbations of IPF, Collard et al. proposed diagnostic criteria for exacerbations and emphasized the need for epidemiological data. ${ }^{3}$ However, there remains little real-world evidence on the incidence rate and the burden of IPFrelated hospitalizations or exacerbations among patients with IPF.

The objectives of this study were to (a) describe the characteristics (e.g., frequency and duration) and (b) estimate incidence rates and costs of all-cause hospitalizations, IPF-related hospitalizations, and possible acute exacerbations in patients diagnosed with IPF in the United States.

\section{Methods}

\section{Data Source}

This retrospective cohort study used commercial administrative claims data dated between January 1, 2006, and December 31, 2011, from the PharMetrics Integrated Database (IMS Lifelink). This database contains administrative claims for approximately 70 million members of approximately 100 managed care organizations from 4 geographical regions (Northeast, Midwest, South, and West) of the United States. Enrollees include commercially insured adults (aged 18-64 years) and Medicare beneficiaries with supplemental insurance (aged $\geq 65$ years).

\section{Patient Selection}

Selected patients were aged $\geq 18$ years, had 12 months of previous continuous health plan enrollment at the time of diagnosis, and were newly diagnosed with IPF between January 1, 2007, and December 31, 2011 (Figure 1). IPF patients were identified as individuals who had at least 2 medical claims with an associated diagnosis of idiopathic fibrosing alveolitis (IFA, International Classification of Disease, Ninth Revision, Clinical Modification [ICD9-CM] code 516.3x) on separate dates, or at least 1 claim with an associated diagnosis of IFA plus at least 1 claim with an associated diagnosis of postinflammatory pulmonary fibrosis (PPF, ICD-9-CM code 515.xx) in any sequence on separate dates. The date of the first claim with IFA was set as the index date. Patients with IPF were further required to have the following: (a) at least 1 claim for lung biopsy or a high-resolution computed tomography (HRCT) of the thorax dated within 90 days before or after the index date; (b) at least 2 claims with IFA after that lung biopsy/HRCT date (and not on the same day as index date); and (c) no claims for other interstitial lung diseases (e.g., connective tissue diseases) after the index date (ICD-9-CM codes are listed in Appendix A, available in online article). Individuals with claims for lung cancer before the index date or with claims for cystic fibrosis during the study period were excluded. Patients were required to have been enrolled for 1 year before the index date. No minimal follow-up duration was required. Patients were followed until 12 months after the index date or until disenrollment from an insurance plan, whichever happened first. 


\section{Study Measures}

Baseline demographics, including age, gender, region, payer type, and plan type were described as of the index date. Comorbidity burden was estimated using the Charlson Comorbidity Index (CCI) for 12 months before the index, which is a widely accepted method to measure comorbidity burden using administrative databases. ${ }^{14-16}$ The CCI assigns a weight (ranging from 1 to 6) according to disease severity for 17 medical conditions to compute an aggregate score, with higher scores indicating greater burden. The comorbidities commonly associated with IPF (e.g., hypertension, emphysema/chronic obstructive pulmonary disease [COPD], coronary artery disease, arrhythmia, and gastroesophageal reflux) according to the American Thoracic Society (ATS) treatment guidelines were assessed during a 1-year pre-index period based on ICD-9-CM codes. ${ }^{10}$

All-cause hospitalizations, IPF-related hospitalizations, possible acute exacerbations requiring hospitalization, and possible acute exacerbations not requiring hospitalization dated within 12 months after the index date were assessed. Due to the lack of radiologic results in the claims database used to clinically confirm IPF exacerbations, we defined possible exacerbations based on place of services and ICD-9-CM codes. IPF-related hospitalizations were defined as hospitalizations with a primary diagnosis of IFA or hospitalizations with a primary diagnosis of respiratory failure (ICD-9-CM codes 786.xx, 518.81, and 518.84) and a secondary diagnosis of IFA. Possible acute exacerbations requiring hospitalization were defined as a subgroup of IPF-related hospitalizations without medical claims for respiratory infection (ICD-9-CM codes 480.xx-488.xx and 010.xx-012.xx); heart failure (ICD-9-CM codes 428.11, 428.21, 428.31, 428.41, 428.13, 428.23, 428.33, and 428.43); pulmonary embolism (ICD-9-CM code 415.xx); or pneumothorax (ICD-9-CM codes 512.xx, 860.xx, and 011.7x) dated within \pm 15 days of the hospital admission date. We analyzed the frequency, incidence rate, duration, and costs of identified hospitalizations, as well as the number of days between the index date and the first all-cause hospitalization, IPF-related hospitalization, or possible acute exacerbation requiring hospitalization. Costs of hospitalization events were inclusive of all medical services received during the hospital stay. Costs were estimated from the third-party payer's perspective, and only the paid amount recorded in the Pharmetrics Integrated Database was used in calculation.

Possible acute exacerbations not requiring hospitalization were defined as emergency room visits or urgent/unscheduled physician visits with a diagnosis of IFA, PPF, or respiratory failure and without diagnoses of respiratory infection, heart failure, pulmonary embolism, or pneumothorax on the same date. Urgent physician visits were identified as outpatient claims with associated Current Procedural Terminology codes (99201-99215 and 99050-99060), Healthcare Common Procedure Coding System codes (S9083 or S9088), or a Centers for Medicare \& Medicaid Services (CMS) place-of-service code (20). Frequency and incidence rate of exacerbations not requiring hospitalization were computed, and associated costs were estimated as the sum of costs of all medical services received on the same date as an emergency room or urgent care visit.

\section{Analysis}

A descriptive analysis was conducted. Baseline demographics and comorbidities were reported for the newly diagnosed IPF patients identified in this study. Mean and standard deviations (SD) were reported for continuous variables. Frequencies and percentages were reported for categorical variables. Characteristics of all-cause or IPF-related hospitalizations and exacerbations were summarized, including frequency, days in hospital, and associated costs. The frequency of all events was calculated for different time windows after the index date: 0-30 days, 31-90 days, 91-180 days, and 181-365 days. Because of variation in duration of follow-up time among the patients with IPF, occurrence of all type of events were summarized as incidence rates per 100 person-years for all patients and for the patients who experienced the events. Costs were inflationadjusted to 2012 U.S. dollars based on the Consumer Price Index published by the U.S. Bureau of Labor Statistics. ${ }^{17}$ Risk of all-cause and IPF-related hospitalizations, along with possible IPF exacerbations requiring or not requiring hospitalization during the 1 year after the index date was plotted using Kaplan-Meier estimates. Further, we estimated the proportion of total IPF-related hospitalization cost to total all-cause hospitalization cost and the proportion of total cost of exacerbation requiring or not requiring hospitalization to total exacerbation cost. Total cost for the specific event was calculated as the average cost per event multiplied by the number of events.

\section{Results}

\section{Baseline Characteristics}

There were 1,735 newly diagnosed patients with IPF identified in this study (Figure 1). Among these patients, 669 (38.6\%) had all-cause hospitalizations; 79 (4.6\%) had possible exacerbations requiring hospitalizations; 1,183 (68.2\%) had possible exacerbations not requiring hospitalizations; and 473 (27.3\%) had not experienced any exacerbations during the follow-up period. The mean $( \pm S D)$ age was $71.5 \pm 12.7$ years, and $54.0 \%$ were male (Table 1). A majority of patients with IPF had commercial insurance $(66.6 \%)$ and were enrolled in preferred provider organization plans (54.7\%). Over $60 \%$ of patients were residents of the South and Midwest regions. On average, patients were followed for $9.7 \pm 3.6$ months from the index date (median=12.0, 25th-75th percentile: 8.0-12.0). The mean CCI score was $2.0 \pm 1.9$. Of the comorbidities, cardiovascular conditions were most common followed by other respiratory diseases, gastroesophageal reflux, depression, and obesity (Table 1). 


\section{TABLE 1}

Baseline Demographics and Clinical Characteristics

\begin{tabular}{|c|c|}
\hline & $\begin{array}{l}\text { All Newly Diagnosed } \\
\text { IPF Patients } \\
(\mathrm{N}=1,735)\end{array}$ \\
\hline Age, mean (SD) & $71.5(12.7)$ \\
\hline \multicolumn{2}{|l|}{ Age categories, years (\%) } \\
\hline$\leq 50$ & 5.1 \\
\hline $51-64$ & 23.9 \\
\hline $65-74$ & 28.0 \\
\hline $75-84$ & 28.4 \\
\hline $85+$ & 14.6 \\
\hline Male (\%) & 54.0 \\
\hline \multicolumn{2}{|l|}{ Region (\%) } \\
\hline Northeast & 23.7 \\
\hline Midwest & 29.3 \\
\hline South & 31.5 \\
\hline West & 15.5 \\
\hline \multicolumn{2}{|l|}{ Payer type at index date (\%) } \\
\hline Commercial & 66.6 \\
\hline Medicaid & 0.1 \\
\hline Medicare & 19.7 \\
\hline Self-insured & 13.5 \\
\hline Missing/unknown & 0.2 \\
\hline \multicolumn{2}{|l|}{ Plan type at index date (\%) } \\
\hline Missing/unknown & 1.3 \\
\hline Consumer directed & 0.6 \\
\hline Health maintenance organizations & 14.0 \\
\hline Indemnity (fee for service) & 16.6 \\
\hline Point of service plan & 12.8 \\
\hline Preferred provider organizations & 54.7 \\
\hline \multicolumn{2}{|l|}{ Follow-up duration (months) } \\
\hline Mean (SD) & $9.7(3.6)$ \\
\hline Median (25th-75th percentile) & $12.0(8.0-12.0)$ \\
\hline \multicolumn{2}{|l|}{ Charlson Comorbidity Index (CCI) } \\
\hline Mean (SD) & $2.0(1.9)$ \\
\hline \multicolumn{2}{|l|}{ CCI category } \\
\hline $\mathrm{CCI}=0$ & 19.9 \\
\hline $\mathrm{CCI}=1$ & 29.0 \\
\hline $\mathrm{CCI}=2$ & 20.5 \\
\hline $\mathrm{CCI}=3$ & 13.1 \\
\hline $\mathrm{CCI} \geq 4$ & 17.5 \\
\hline \multicolumn{2}{|c|}{ Common IPF-associated comorbidities (\%) } \\
\hline Pulmonary hypertension & 5.5 \\
\hline Hypertension & 66.7 \\
\hline Gastroesophageal reflux & 23.7 \\
\hline Obstructive sleep apnea & 11.5 \\
\hline Obesity & 6.5 \\
\hline Emphysema/COPD & 40.2 \\
\hline Depression & 10.4 \\
\hline Asthma & 17.6 \\
\hline Arrhythmia & 27.6 \\
\hline CAD & 38.4 \\
\hline
\end{tabular}

$C A D=$ coronary artery disease $; C O P D=$ chronic obstructive pulmonary disease; $I P F=$ idiopathic pulmonary fibrosis; $S D=$ standard deviation .

\section{All-Cause Hospitalizations}

Of the study population, 1,174 hospitalizations were accumulated by 669 (38.6\%) patients within a 1-year post-index period. The overall incidence rate was 83 admissions per 100 person-years among all IPF patients and 228 admissions per 100 person-years among the patients with events (Table 2). The most common primary diagnosis was PPF (number of admissions: 162), which accounted for $13.8 \%$ of all the hospitalizations, followed by pneumonia (number of admissions: 60, 5.1\%) and IFA (number of admissions: 45, 3.8\%; Appendix $\mathrm{B}$, available in online article). On average, patients stayed in the hospital for $9(\mathrm{SD} \pm 11)$ days with a mean $( \pm \mathrm{SD})$ cost of $\$ 13,987 \pm \$ 41,988$ and median (25th-75th percentile) cost of $\$ 4,690(\$ 1,158-\$ 12,108)$ per hospital stay (Table 2).

\section{IPF-Related Hospitalizations}

A total of 234 IPF-related hospitalizations were accumulated by $188(10.8 \%)$ patients during a 1-year post-index period, with 17 IPF-related admissions per 100 person-years (Table 2). The incidence rate was 174 admissions per 100 person-years among the patients with events. The average length of stay was $8 \pm 10$ days with an average cost $( \pm$ SD) of $\$ 16,812 \pm \$ 66,399$ and median (25th-75th percentile) cost of $\$ 3,914$ (\$1,469-\$12,096) per hospital stay.

Among the 234 IPF-related hospitalizations, less than half $(n=102)$ met the criteria for exacerbations requiring hospitalization. Of the remaining 132 IPF-related hospitalizations not classified as exacerbations, there were 94 hospitalizations (69.6\%) with at least 1 medical claim for respiratory infection within 15 days of the admission, 53 (22.3\%) claims for pneumothorax, 20 (14.8\%) claims for heart failure, and 11 (8.1\%) claims for pulmonary embolism.

\section{Possible Exacerbations Requiring Hospitalizations}

There were 102 possible exacerbations requiring hospitalization identified among 79 (4.6\%) patients with IPF for an estimated overall incidence rate of 7 per 100 person-years. The incidence rate was 168 admissions per 100 person-years among the patients with events. On average, patients with an exacerbation requiring hospitalization stayed in hospital for $6 \pm 7$ days with an average cost $( \pm$ SD) of $\$ 14,731 \pm 85,468$ and median (25th-75th percentile) cost of $\$ 2,213(\$ 1,469-\$ 6,657)$ per hospital stay (Table 2).

\section{Possible Exacerbations Not Requiring Hospitalizations}

During the 1-year post-index period, 3,903 possible exacerbations not requiring hospitalization were identified among 1,251 (72.1\%) patients, for an estimated incidence rate of 277 episodes per 100 person-years. The incidence rate was 365 events per 100 person-years among the patients with events. For these events, mean $( \pm S D)$ cost was $\$ 444 \pm \$ 1,481$ and median (25th-75th percentile) cost was $\$ 176$ (\$96-\$380) per visit (Table 2). 
TABLE 2 Frequency, Duration, and Costs of Hospitalizations and Exacerbations During 1 -Year Post-index Period $(\mathrm{N}=1,735)$

\begin{tabular}{|c|c|c|c|c|}
\hline & $\begin{array}{c}\text { All-Cause } \\
\text { Hospitalizations }\end{array}$ & $\begin{array}{c}\text { All IPF-Related } \\
\text { Hospitalizations }\end{array}$ & $\begin{array}{c}\text { Exacerbations Requiring } \\
\text { Hospitalization }\end{array}$ & $\begin{array}{c}\text { Exacerbations Not } \\
\text { Requiring Hospitalization }^{a} \\
\end{array}$ \\
\hline Patients with at least 1 event, n (\%) & $669(38.6)$ & $188(10.8)$ & $79(4.6)$ & $1,251(72.1)$ \\
\hline Rate per 100 person-years $(95 \% \mathrm{CI})$ & $83(79-88)$ & $17(14-19)$ & $7(6-9)$ & $277(269-286)$ \\
\hline $\begin{array}{l}\text { Rate per } 100 \text { person-years among } \\
\text { patients with events }(95 \% \mathrm{CI})\end{array}$ & $228(215-241)$ & $174(151-196)$ & $168(136-201)$ & $365(354-377)$ \\
\hline Number of events within 1 year & 1,174 & 234 & 102 & 3,903 \\
\hline \multicolumn{5}{|l|}{ Days in hospital per event } \\
\hline Mean (SD) & $9(11)$ & $8(10)$ & $6(7)$ & NA \\
\hline Median (25th-75th percentile) & $5(3-11)$ & $5(3-9)$ & $4(3-8)$ & NA \\
\hline \multicolumn{5}{|l|}{ Costs per event (\$) } \\
\hline Mean (SD) & $13,987(41,988)$ & $16,812(66,399)$ & $14,731(85,468)$ & $444(1,481)$ \\
\hline Median & 4,690 & 3,914 & 2,213 & 176 \\
\hline 25th-75th percentile & $1,158-12,108$ & $1,469-12,096$ & $1,469-6,657$ & $96-380$ \\
\hline
\end{tabular}

aSubgroups are not mutually exclusive; Exacerbations Not Requiring Hospitalization column may include patients with exacerbation requiring hospitalization.

$C I=$ confidence interval; $I P F=$ idiopathic pulmonary fibrosis; $N A=$ not applicable; $S D=$ standard deviation.

\section{The Frequency and Risk of Hospitalizations and Possible Exacerbations}

The occurrence of events was most common within the first month of the index date. During this period, $16.5 \%$ of patients were admitted to the hospital; 5.3.\% were hospitalized due to IPF; $2.4 \%$ experienced a possible exacerbation requiring hospitalization; and over $50 \%$ had a possible exacerbation not requiring hospitalization. The frequency of all 4 events decreased during the time windows of 31-90 days, 91-180 days, and 181-365 days compared with that of the first month (Figure 2).

Risk of all-cause and IPF-related hospitalizations and possible IPF exacerbations requiring or not requiring hospitalization are depicted in Figure 3. The cumulative risk of each event increased over time.

\section{Total Hospitalization Cost and Total Exacerbation Cost}

For this study cohort, total IPF-related hospitalization cost is estimated at over $\$ 3.9$ million $(\$ 16,812$ per stay $\times 234$ hospital stays) accounting for $23.96 \%$ of total all-cause hospitalization cost, which is estimated to be more than $\$ 16$ million $(\$ 13,987$ per stay $\times 1,174$ hospital stays). As much as $\$ 3.2$ million incurred by this group of IPF patients was associated with possible IPF exacerbation events; the cost of exacerbations requiring hospitalizations is over $\$ 1.5$ million ( $\$ 14,731$ per stay $\times 102$ hospital stays), accounting for $46.44 \%$ of the total exacerbation associated cost, and the cost of exacerbation not requiring hospitalizations accounts for $53.56 \%$, reaching $\$ 1.7$ million, because of its high occurrence $(n=3,903)$.

\section{Discussion}

A high proportion of patients with newly diagnosed IPF were found to have at least 1 hospitalization or a possible exacerbation not requiring hospitalization in the first year after IPF diagnosis. The majority of possible exacerbations occurred within the first 90 days after the IPF diagnosis. Limited data related to IPF hospitalization or exacerbations are found in the literature. To our knowledge, this is the first observational study to assess frequency, incidence rate, and costs for different types of health care resource utilization among patients with IPF. Among this IPF cohort, we found the rate of all-cause hospitalization (83 per 100 person-years) comparable with the rate of all-cause hospitalizations among elderly Medicare patients with COPD (107 per 100 person-years). ${ }^{18}$ Costs of exacerbations observed in patients with newly diagnosed IPF were almost double compared with patients with COPD. A recent study of a U.S. managed care population reported mean costs $( \pm$ SD) per exacerbation among patients with COPD as $\$ 8,219 \pm \$ 22,644$ or $\$ 269 \pm \$ 748$ if exacerbations did not result in hospitalization. ${ }^{19}$ The comparison with COPD exacerbation indicates a high burden incurred on IPF patients and the health care system caused by hospitalizations and exacerbations.

In the 2011 ATS/ERS/JRS/ALAT IPF guidelines (a collaborative effort of the American Thoracic Society, the European Respiratory Society, the Japanese Respiratory Society, and the Latin American Thoracic Association), the estimated annual incidence of acute exacerbation from all causes among patients with IPF was summarized as 5\%-10\%. ${ }^{7-10}$ Estimates for exacerbations have been reported as high as $23.9 \%$ over a 3 -year period. ${ }^{12}$ However, none of these estimates differentiated between exacerbations that required hospitalization and those that did not. Furthermore, those studies included prevalent and incident IPF cases. Patients included in recent clinical trials were required to be diagnosed within 5 years ${ }^{20}$; consequently, with a median survival of 3 years, they represent a large group of survivors with IPF. In contrast, our study was 


\section{FIGURE 2 Proportion of Patients with Hospitalizations and Possible Exacerbations} Within Different Time Windows Since Index Date

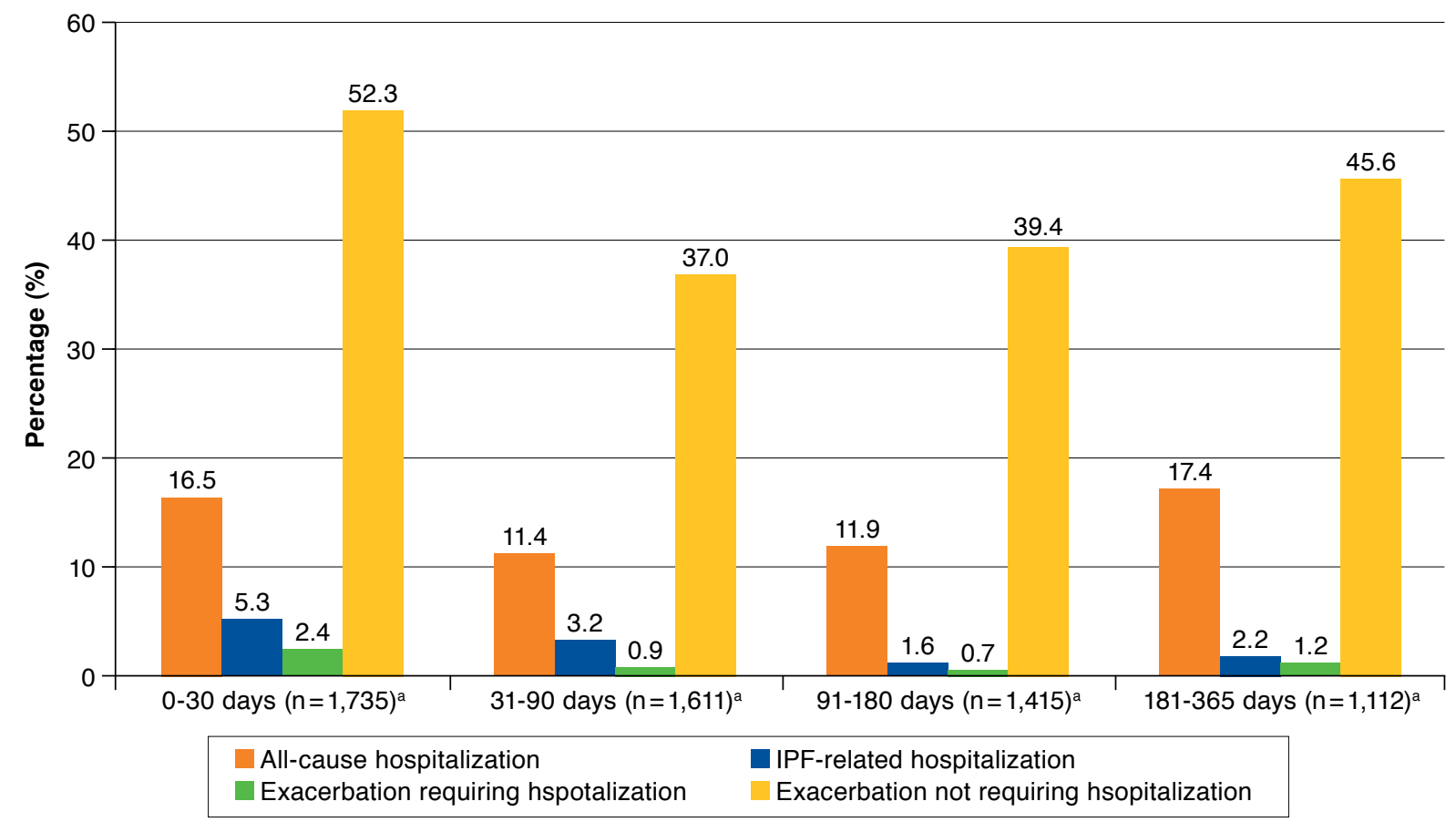

${ }^{a}$ Only the patients with enrollment for the respective time window were included. $I P F=$ idiopathic pulmonary fibrosis.

limited to incident IPF cases only, representing patients who are actively coping and adapting to their new diagnosis, which may result in more use early in the course of their illness. Moreover, incident cases have more diverse disease trajectories, including a subgroup of patients with higher mortality, that may also partially explain the higher occurrence of events within the first 90 days of the index IPF diagnosis.

High incidence rates and average costs were noted for hospitalizations and exacerbations among patients with IPF, suggesting that these factors are likely to be important components in the incurrence of substantial burden to patients as well as the overall health system. This is consistent with previous studies that show high prevalence of hospitalizations and exacerbations associated with IPF, which may potentially contribute to an increase in overall health care resource utilization and costs among patients with IPF. ${ }^{13,21}$ Collard et al. (2012, 2015) found that when compared with age-, gender-, race-, and region-matched controls, patients with IPF had a 134\% higher risk of hospitalization (per person-year) and a 126\% increased risk of emergency room visits (per person-year); the total direct costs for patients with IPF were $\$ 20,887$ per person-year (2011 value). ${ }^{22,23}$ Furthermore, previous studies have shown that exacerbations and hospitalization represent clinically meaningful events in patients with IPF., 5,24

The definition and diagnosis of acute IPF exacerbation is a challenge and has not been standardized at the individual patient level in either the research or the clinical setting. The INPULSIS trials showed that in the clinical trial setting, exacerbations are infrequent events in patients with $\mathrm{IPF}^{20}$ This study is offering a complementary approach that may be useful for surveillance of exacerbations of IPF at the population level. ${ }^{25}$ In addition, the literature is lacking with regards to the cost of hospitalizations and exacerbations in an IPF population, and this study helps to fill the evidence gap to provide insights on economic burden of hospitalization and possible exacerbations among patients diagnosed with IPF.

High unmet medical needs exist for IPF management. In past practice, a common treatment was a combination of prednisone, azathioprine, and N-acetylcysteine. However, in the PATHER trial, this treatment has been proven ineffective and harmful to patients. ${ }^{26}$ The therapies newly approved by the U.S. Food and Drug Administration were recently included in updates of the ATS/ERS/JRS/ALT IPF guidelines as conditional recommendations for IPF treatment and may offer hope for patients with this 


\section{FIGURE 3 Risk of All-Cause and IPF-Related Hospitalizations and Possible Acute IPF Exacerbations Requiring} and Not Requiring Hospitalization During 1-Year Post-index Period

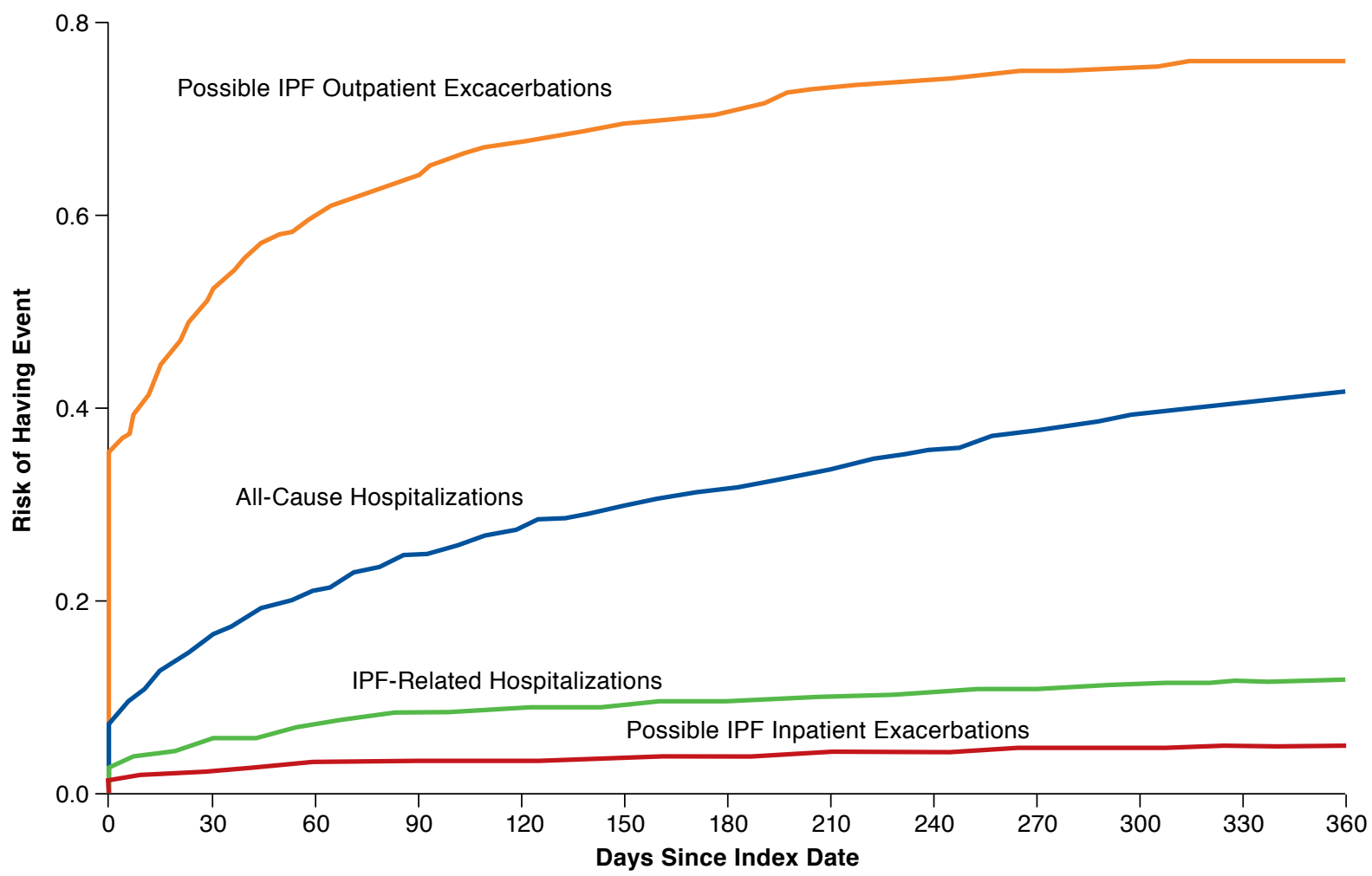

IPF = idiopathic pulmonary fibrosis.

serious and possibly life-threatening condition. ${ }^{27}$ Future studies should be considered to better understand the effectiveness of these newly emerging therapies in real-world practice.

\section{Limitations}

Results of this study need to be interpreted in the light of several limitations, some of which are common to retrospective database analyses. The health care claims data in this analysis were primarily used for administrative purposes to obtain reimbursement for services provided to health plan members. As a result, diagnostic and procedural coding inaccuracies may occur, causing misclassification of certain diagnoses, visits, or procedures. To minimize potential diagnostic misclassification, a strict algorithm was used requiring diagnosis codes relevant to IPF and procedure codes for HRCT or lung biopsy, as well as at least 2 additional claims with a diagnosis of IFA after the HRCT or lung biopsy for confirmatory purposes. Moreover, a 12-month pre-index continuous enrollment was required to ensure complete patient data in order to examine baseline characteristics and to define incident IPF patients. In addition, patients were excluded if they had claims for diagno- ses of other interstitial lung diseases after the first claim with a diagnosis of IFA. The same diagnosis codes but less stringent identification methods have been used in published retrospective claims-based studies reported by Collard et al. and Raghu et al. ${ }^{22,28}$ Furthermore, the distribution of men and women in this cohort (53.9\% vs. $46.1 \%)$ is similar to these previous reports, ${ }^{22,28}$ which provides further validation of the approach used to identify incident cases.

In order to improve the accuracy of possible IPF exacerbation episodes identified using claims data, we adopted the exacerbation definition described by Collard et al. $^{3}$ and excluded hospitalization, emergency room visits, and urgent care physician visits with medical claims indicating reasons (based on diagnosis) other than IFA, PPF, or respiratory failure, such as respiratory infection, heart failure, pulmonary embolism, or pneumothorax dated within \pm 15 days of a hospital admission date. Because of the lack of clinical information in administrative claims data, complete differentiation between acute respiratory worsening and acute exacerbations of IPF is not possible, even after applying the aforementioned algorithm. 
This may partly explain the relatively high rate of possible exacerbations not requiring hospitalization compared with the incidence of exacerbations reported in the existing literature. However, the rate of possible exacerbation requiring hospitalizations is in line with the reported evidence. Furthermore, unlike clinical trials or studies that use medical records, it is difficult to differentiate the sequence of exacerbations or infections using only administrative claims, since they may be coded on the same date when patients visited their physicians. Therefore, some of the possible exacerbations not requiring hospitalization identified in this study could be acute respiratory worsening and might not be classified as an exacerbation in the setting of a clinical trial or with a medical chart review.

Most individuals included in PharMetrics data were enrolled in either commercial plans or supplemental Medicare plans. Therefore, Medicare patients with traditional fee-for-service benefits were underrepresented, ${ }^{29}$ and the results may not be generalizable to this population. In addition, hospitalization of elderly patients in this study may not have been fully captured because their inpatient claims may have been submitted to CMS for processing and not captured by their managed care plans. Therefore, the incidence of all-cause hospitalization and possible exacerbations resulting in hospitalization may be underestimated for elderly patients.

\section{Conclusions}

$\overline{\text { All-cause hospitalizations and possible IPF exacerbations were }}$ associated with high economic burden, which was driven mainly by the cost of hospitalization and the high occurrence of possible exacerbations not requiring hospitalization. These results suggest a need for effective treatments to slow disease progression among patients with IPF.

\section{Authors}

YANNI F. YU, DSc, is Product Director, Health Economics and Outcomes Research, Boehringer Ingelheim Pharmaceuticals, Ridgefield, Connecticut; GIOVANNA DEVERCELLI, PhD, is Senior Director, Shire Pharmaceuticals, Lexington, Massachusetts; NING WU, PhD, is HEOR Contractor, Biogen, Cambridge, Massachusetts; CHIEN-CHIA CHUANG, PhD, is Senior Manager, Sunovion Pharmaceuticals, Marlborough, Massachusetts; and DAVID B. COULTAS, MD, is Chief of Staff, Veterans Affairs Portland Health Care System, Portland, Oregon. ROSA WANG, BS, MHA, is Research Associate; XIAOYUN PAN, MS, PhD, is Research Scientist; and NICOLE N. BENJAMIN, BS, is Consultant, Evidera, Lexington, Massachusetts..

AUTHOR CORRESPONDENCE: Xiaoyun Pan, MS, PhD, Research Scientist, Evidera, 430 Bedford St., Ste. 300, Lexington, MA 02420. Tel.: 781.960.0317; E-mail: lucy.pan@evidera.com.

\section{DISCLOSURES}

Boehringer Ingelheim Pharmaceuticals, Inc. (BIPI) provided funding for this study. Yu and Devercelli are currently salaried employees of BIPI. Wu, Chuang, Wang, Pan, and Benjamin are currently employees of Evidera, which provides consulting and other research services to pharmaceutical, device, government, and nongovernment organizations. In their salaried positions, they work with a variety of companies and organizations and are precluded from receiving payment or honoraria directly from these organizations for services rendered. Evidera received funding from BIPI to conduct the analysis. Coultas was previously a paid consultant of BIPI. The contents do not represent the views of the Department of Veterans Affairs or the U.S. government.

This manuscript does not contain clinical studies or patient data. The authors have full control of all primary data, and they agree to allow the journal to review their data if requested.

All authors meet the criteria for authorship as recommended by the International Committee of Medical Journal Editors, and they are fully responsible for all content and editorial decisions and were involved at all stages of manuscript development.

The manuscript was drafted by Benjamin, $\mathrm{Wu}$, and $\mathrm{Yu}$ and revised by Wang, Pan, Yu, Coultas, and Devercelli. The study was designed by $\mathrm{Yu}, \mathrm{Wu}$, Chuang, Wang, Benjamin, and Coultas. Statistical analysis was conducted by $\mathrm{Wu}$, Chuang, and Wang. Senior review was provided by Coultas and Devercelli.

\section{REFERENCES}

1. Gulati M. Diagnostic assessment of patients with interstitial lung disease. Prim Care Respir J. 2011;20(2):120-27.

2. Selman M, Carrillo G, Estrada A, et al. Accelerated variant of idiopathic pulmonary fibrosis: clinical behavior and gene expression pattern. PLoS One. 2007;30;2(5):e482.

3. Collard HR, Moore BB, Flaherty KR, et al. Acute exacerbations of idiopathic pulmonary fibrosis. Am J Respir Crit Care Med. 2007;176(7):636-43.

4. Agarwal R, Jindal SK. Acute exacerbation of idiopathic pulmonary fibrosis: a systematic review. Eur J Intern Med. 2008;19(4):227-35.

5. Collard HR, Yow E, Richeldi L, Anstrom KJ, Glazer C; IPFnet Investigators. Suspected acute exacerbation of idiopathic pulmonary fibrosis as an outcome measure in clinical trials. Respir Res. 2013;14:73.

6. Kubo H, Nakayama K, Yanai M, et al. Anticoagulant therapy for idiopathic pulmonary fibrosis. Chest. 2005;128(3):1475-82.

7. Kim DS. Acute exacerbations in patients with idiopathic pulmonary fibrosis. Respir Res. 2013;14:86

8. Azuma A, Nukiwa T, Tsuboi E, et al. Double-blind, placebo-controlled trial of pirfenidone in patients with idiopathic pulmonary fibrosis. Am J Respir Crit Care Med. 2005;171(9):1040-47.

9. Kim DS, Park JH, Park BK, Lee JS, Nicholson AG, Colby T. Acute exacerbation of idiopathic pulmonary fibrosis: frequency and clinical features. Eur Respir J. 2006;27(1):143-50

10. Raghu G, Collard HR, Egan JJ, et al. An official ATS/ERS/JRS/ALAT statement: idiopathic pulmonary fibrosis: evidence-based guidelines for diagnosis and management. Am J Respir Crit Care Med. 2011;183(6):788-824.

11. Kim DS. Acute exacerbation of idiopathic pulmonary fibrosis. Clin Chest Med. 2012;33(1):59-68

12. Kondoh Y, Taniguchi H, Katsuta T, et al. Risk factors of acute exacerbation of idiopathic pulmonary fibrosis. Sarcoidosis Vasc Diffuse Lung Dis. 2010;27(2):103-10.

13. Song JW, Hong SB, Lim CM, Koh Y, Kim DS. Acute exacerbation of idiopathic pulmonary fibrosis: incidence, risk factors and outcome. Eur Respir J. 2011;37(2):356-63.

14. Deyo RA, Cherkin DC, Ciol MA. Adapting a clinical comorbidity index for use with ICD-9-CM administrative databases. J Clin Epidemiol. 1992:45(6):613-19. 
15. D'Hoore W, Bouckaert A, Tilquin C. Practical considerations on the use of the Charlson comorbidity index with administrative data bases. J Clin Epidemiol. 1996;49(12):1429-33.

16. Schneeweiss S, Maclure M. Use of comorbidity scores for control of confounding in studies using administrative databases. Int J Epidemiol. 2000;29(5):891-98

17. Marshall WJ, Bangert SK. Clinical Biochemistry: Metabolic and Clinical Aspects. 2nd ed. London: Churchill Livingstone; 2008.

18. Baillargeon J, Wang Y, Kuo YF, Holmes HM, Sharma G. Temporal trends in hospitalization rates for older adults with chronic obstructive pulmonary disease. Am J Med. 2013;126(7):607-14.

19. Abudagga A, Sun SX, Tan H, Solem CT. Exacerbations among chronic bronchitis patients treated with maintenance medications from a US managed care population: an administrative claims data analysis. Int J Chron Obstruct Pulmon Dis. 2013;8:175-85.

20. Richeldi L, du Bois RM, Raghu G, et al. Efficacy and safety of nintedanib in idiopathic pulmonary fibrosis. N Engl J Med. 2014;370(22):2071-82.

21. Brown AW, Fischer CP, Shlobin OA, et al. Outcomes after hospitalization in idiopathic pulmonary fibrosis: a cohort study. Chest. 2015;147(1):173-79.

22. Collard HR, Ward AJ, Lanes S, Cortney Hayflinger D, Rosenberg DM, Hunsche E. Burden of illness in idiopathic pulmonary fibrosis. J Med Econ. 2012;15(5):829-35.
23. Collard HR, Chen SY, Yeh WS, et al. Health care utilization and costs in idiopathic pulmonary fibrosis in U.S. Medicare beneficiaries aged 65 years and older. Ann Am Thorac Soc. 2015;12(7):981-87.

24. Groenewegen KH, Schols AM, Wouters EF. Mortality and mortalityrelated factors after hospitalization for acute exacerbation of COPD. Chest. 2003;124(2):459-67.

25. Institute of Medicine (IOM). A Nationwide Framework for Surveillance of Cardiovascular and Chronic Lung Diseases. Washington, DC: National Academies Press; 2011.

26. Evaluating the effectiveness of prednisone, azathioprine, and n-acetylcysteine in patients with IPF (PANTHER-IPF). Identifier: NCT00650091. ClinicalTrials.gov. 2015. Available at: https://clinicaltrials. gov/ct2/show/NCT00650091. Accessed January 21, 2016.

27. Raghu G, Rochwerg B, Zhang Y, et al. An Official ATS/ERS/JRS/ALAT Clinical Practice Guideline: Treatment of Idiopathic Pulmonary Fibrosis. An update of the 2011 clinical practice guideline. Am J Respir Crit Care Med. 2015;192(2):e3-19.

28. Raghu G, Weycker D, Edelsberg J, Bradford WZ, Oster G. Incidence and prevalence of idiopathic pulmonary fibrosis. Am J Respir Crit Care Med. 2006;174(7):810-16.

29. Sun P, Seftel A, Swindle R, Ye W, Pohl G. The costs of caring for erectile dysfunction in a managed care setting: evidence from a large national claims database. J Urol. 2005;174(5):1948-52. 


\begin{tabular}{|c|c|}
\hline APPENDIX A & $\begin{array}{l}\text { ICD-9-CM Diagnosis Codes and } \\
\text { CPT-4 Codes Applied in the Sample } \\
\text { Selection }\end{array}$ \\
\hline Disease/Procedure & Codes \\
\hline IPF & ICD-9-CM: $516.3 x$ \\
\hline PPF & ICD-9-CM: 515.xx \\
\hline Lung biopsy & $\begin{array}{l}\text { ICD-9-CM: 33.28, 34.21; CPT-4: 32095, 32100- } \\
\text { 32160, 32400-32405, 32095-32098, 31622-31651, } \\
\text { 32601-32674 }\end{array}$ \\
\hline HRCT & ICD-9-CM: 87.41; CPT-4: 71250-71270 \\
\hline Interstitial lung disease & $\begin{array}{l}\text { ICD-9-CM: 135, 162.x, 237.7x, 272.7, 277.3x, } \\
\text { 277.8x, 446.21, 446.4, 495.x, 500, 501, 502, } \\
503,504,505,506.4,508.1,508.8,516.0,516.1 \\
516.2,516.8,516.9,517.2,517.8,518.3,555 . x \\
710.0,710.1,710.2,710.3,714.4,714.81,720 . x \\
759.5\end{array}$ \\
\hline Lung cancer & ICD-9-CM: 162.xx, 197.0 \\
\hline Cystic fibrosis & ICD-9-CM: 277.0 \\
\hline \multicolumn{2}{|c|}{$\begin{array}{l}\text { CPT = Current Procedural Terminology; HRCT = High-resolution computed tomog- } \\
\text { raphy; ICD-9-CM = International Classification of Diseases, Ninth Revision, } \\
\text { Clinical Modification; IPF = idiopathic pulmonary fibrosis; PPF = postinflammatory } \\
\text { pulmonary fibrosis. }\end{array}$} \\
\hline
\end{tabular}

\begin{tabular}{l|l|l|c|c}
\hline \multicolumn{4}{c}{ APPENDIX B } & $\begin{array}{l}\text { Most Common Primary Diagnoses } \\
\text { of Hospitalizations in 1-Year } \\
\text { Post-index Period (Total } \\
\text { Hospitalization Events = 1, 174) }\end{array}$ \\
\hline Rank & $\begin{array}{c}\text { ICD-9-CM } \\
\text { Code }\end{array}$ & \multicolumn{1}{|c}{ Description } & Frequency & Percentage \\
\hline 1 & 515 & $\begin{array}{l}\text { Postinflammatory pulmonary } \\
\text { fibrosis }\end{array}$ & 162 & 13.8 \\
\hline 2 & 486 & $\begin{array}{l}\text { Pneumonia, organism } \\
\text { unspecified }\end{array}$ & 60 & 5.1 \\
\hline 3 & 516.3 & Idiopathic fibrosing alveolitis & 45 & 3.8 \\
\hline 4 & V57.89 & $\begin{array}{l}\text { Other specified rehabilitation } \\
\text { procedure }\end{array}$ & 43 & 3.7 \\
\hline 5 & 518.81 & Respiratory failure & 42 & 3.6 \\
\hline 6 & 428 & Congestive heart failure & 34 & 2.9 \\
\hline 7 & 491.21 & $\begin{array}{l}\text { Obstructive chronic bronchitis } \\
\text { with exacerbations }\end{array}$ & 27 & 2.3 \\
\hline 8 & 427.31 & Atrial fibrillation & 26 & 2.2 \\
\hline 9 & 389 & Unspecified septicemia & 15 & 1.3 \\
\hline 10 & 410.71 & $\begin{array}{l}\text { Subendocardial infarction, } \\
\text { initial episode of care }\end{array}$ & 15 & 1.3 \\
\hline
\end{tabular}

ICD-9-CM=International Classification of Diseases, Ninth Revision, Clinical Modification. 